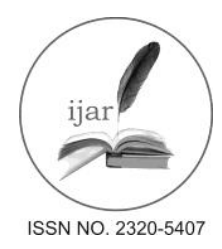

Journal homepage: http://www.journalijar.com

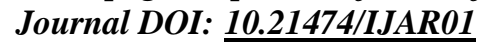

INTERNATIONAL JOURNAL

OF ADVANCED RESEARCH

RESEARCH ARTICLE

\title{
CASE REPORT: DOEGE-POTTER SYNDROME.
}

\author{
Dr. Kishor D. Kharche ${ }^{1}$, Dr. Kavitha Mandapati ${ }^{1}$, Dr. Aditya Hegde ${ }^{1}$, Dr. Dipthi ${ }^{1}$, Dr. Santosh $\mathbf{B}^{1}$, \\ Dr. P. Sudhakar Reddy ${ }^{2}$, Dr. Raman Boddula ${ }^{2}$ \\ 1. Resident, Department of Endocrinology, Yashoda Hospital, Secunderabad. \\ 2. Senior Consultant, Department of Endocrinology, Yashoda Hospital, Secunderabad.
}

\section{Manuscript Info}

Manuscript History:

Received: 18 March 2016

Final Accepted: 29 April 2016

Published Online: May 2016

Key words:

Doege Potter syndrome, Nonislet cell tumour hypoglycemia, Solitary fibrous tumour of pleura.

*Corresponding Author

Dr. Kishor D. Kharche.

\section{Abstract}

Introduction:- Solitary fibrous tumour of the pleura (SFTP) is a rare pleural tumour. Sometimes, these tumours can produce a large IGF-2 molecule which causes hypoglycemic episodes which is referred to as Doege Potter syndrome (DPS). Doege Potter syndrome can present with life-threatening hypoglycemia, hence its early recognition is important as many are amenable to surgical resection. We report a case of benign SFTP presenting with Doege Potter syndrome, that presented to our hospital in 2014.

Case Presentation:- A 45 year old women with history of intra-thoracic tumour presented with recurrent hypoglycemia. At the time of hypoglycemia (blood sugar $32 \mathrm{mg} / \mathrm{dl}$ ), S. Insulin $<0.5 \mathrm{uU} / \mathrm{ml}$ and C-peptide $=0.699 \mathrm{ng} / \mathrm{ml}$. IGF-1 was $<25 \mathrm{ng} / \mathrm{ml}$ and IGF-2 was $328 \mathrm{ng} / \mathrm{ml}$. IGF2:IGF1 ratio was $>13$. CECT Chest showed a large well defined smooth marginated, hypodense, soft tissue mass lesion of size $20 \times 12 \times 13 \mathrm{cms}$ with mild heterogeneous enhancement seen in mid and lower zones of right lung. CT guided biopsy of tumor was suggestive of spindle cell tumor of pleura. Immunohistochemistry showed a bland spindle cell neoplasm in collagenised stroma. Tumor expessed CD 34, Mic 2, bcl 2 and was immune-reactive for cytokeratin, EMA, Calretinin, Calponin, CD 56. PET scan showed a mild metabolically active large right lung lesion with no tracer activity in liver, adrenal or other sites. Thus, a diagnosis of a benign SFTP with Doege Potter syndrome was made. Hypoglycemic management of our patient included regular IV dextrose infusion, scheduled, intermittent feeding overnight and IV steroids. The patient, then underwent surgical resection of the tumor. During the postoperative period, she had no hypoglycemic episodes, IGF-1 was $25 \mathrm{ng} / \mathrm{ml}$ and IGF-2 was $196 \mathrm{ng} / \mathrm{ml}$, and the IGF-2:IGF-1 ratio came down $(<10)$. Patient discharged in stable condition without any hypoglycemia or any need of steroids.

Conclusion:- Doege Potter syndrome is a paraneoplastic manifestation of large non-islet tumour due to production of IGF-2. It should be considered in patients who have intra-thoracic tumour and recurrent hypo-insulinemic hypoglycemia. Most case series on pleural fibrous tumours report hypoglycemia in about 2-4\%. The ratio IGF-2 to IGF-1 is used as a surrogate marker to diagnose Doege Potter syndrome. Surgical resection of the tumour is the treatment modality of choice, which cures the hypoglycemia. 


\section{Introduction:-}

Solitary fibrous tumour of the pleura (SFTP) is one of the rare primary tumours, constituting about $5 \%$ of all pleural tumours [1]. Sometimes, these tumours can produce a large IGF-2 molecule which causes hypoglycemic episodes. This paraneoplastic manifestation of SFTP is referred to as Doege Potter syndrome (DPS) [2]. IGF-2 is a factor stimulating normal cellular growth and proliferation and is also recognized a possible mitogen in pathogenesis of number of neoplasms [3]. Doege Potter syndrome can present with life-threatening hypoglycemia, hence its early recognition is important as many are amenable to surgical resection.

We report a case of benign SFTP presenting with Doege Potter syndrome, that presented to our hospital in 2014.

\section{Case Presentation:-}

A 45 year old women presented with history of sudden onset of weakness of left side of the body associated with altered sensorium. She had history of repeated episodes of sweating, palpitations and heaviness in the chest in the last 3 months, which were occasionally associated with altered sensorium which recovered after taking sugar solution. These symptoms were more common in the early hours of the day. She was a known hypertensive and nondiabetic. She was diagnosed with intra-thoracic tumour about 5 days back.

In the emergency room, she was drowsy and had left sided CVA. Vitals were stable, diaphoresis present. Breath sounds were reduced in the right inter-scapular and infra-scapular areas, no other significant clinical findings. Her GRBS was $37 \mathrm{mg} / \mathrm{dl}$ and she was given IV dextrose, following which is regained her consciousness. There was neither history of any drugs that might cause hypoglycemia nor starvation. Her liver function tests, renal function tests, thyroid profile and serum cortisol were normal. MRI brain showed hyperacute infarct in the anterior centrum semiovale in the right posterior frontal region.

But even with regular infusion of $10 \%$ Dextrose, her blood sugar levels were falling precipitously. Hence she was further evaluated for recurrent hypoglycemia. At the time of hypoglycemia (blood sugar $32 \mathrm{mg} / \mathrm{dl}$ ) serum insulin and $\mathrm{C}$ peptide were low [S. Insulin $<0.5 \mathrm{uU} / \mathrm{ml}(2.60-37.60 \mathrm{uU} / \mathrm{ml})$ and C-peptide $=0.699 \mathrm{ng} / \mathrm{ml}(0.9-7.1 \mathrm{mg} / \mathrm{ml})]$. Due to the low levels of insulin and C-peptide during hypoglycemic episode, hyper-insulinemic hypoglycemia was ruled out (i.e secondary to insulinoma, ectopic insulin production or exogenous insulin administration) and Nonislet cell tumour hypoglycemia $(\mathrm{NICTH})$ was suspected as the patient was known to have a intra-thoracic tumour. Serum IGF-1 and IGF-2 were sent [IGF-1 was $<25 \mathrm{ng} / \mathrm{ml} \mathrm{(101-267} \mathrm{ng/ml} \mathrm{for} \mathrm{age} \mathrm{41-45} \mathrm{years)} \mathrm{and} \mathrm{IGF-2}$ was $328 \mathrm{ng} / \mathrm{ml}(288-736 \mathrm{ng} / \mathrm{ml})$ ). IGF2:IGF1 ratio was $>13$. (3:1 is normal, and $>10$ has been suggested to be pathognomonic for NICTH[4]. Chest X ray PA view showed a large soft tissue mass lesion involving right midzone and lower zone with obscured costo-phrenic angles, causing mediastinal shift to left side. CECT Chest showed a large well defined smooth marginated, hypo dense, soft tissue mass lesion of size 20x12x13 cms with mild heterogeneous enhancement seen in mid and lower zones of right lung. CT guided biopsy of tumor was suggestive of spindle cell tumor of pleura (fibrous tumor). Immunohistochemistry showed a bland spindle cell neoplasm in collagenised stroma. Tumor expessed CD 34, Mic 2, bcl 2 and was immune-reactive for cytokeratin, EMA, Calretinin, Calponin, CD 56. PET scan was done and it showed a mild metabolically active large right lung lesion with no tracer activity in liver, adrenal or other sites. Thus, a diagnosis of a benign SFTP with Doege Potter syndrome was made. Review of the literature was done and surgical resection of the tumour was planned. However, control of repeated hypoglycemic events in the run-up to the surgery was not easy.

Management of Doege-Potter syndrome in our patient was focused on maintaining euglycemia throughout the day. It included regular IV dextrose infusion and scheduled, intermittent feeding overnight. Patient was also started on IV steroids to control hypoglycemia. The patient, then underwent surgical resection of the tumor. En-bloc resection of the tumour was done. During the postoperative period, she had no hypoglycemic episodes, IGF-1 was $25 \mathrm{ng} / \mathrm{ml}$ and IGF-2 was $196 \mathrm{ng} / \mathrm{ml}$, and the IGF-2:IGF-1 ratio came down $(<10)$. Patient discharged in stable condition without any hypoglycemia or any need of steroids.

\section{Discussion:-}

Doege Potter syndrome is a paraneoplastic manifestation of large non-islet tumour. Association of intra-thoracic tumour with hypoglycemia was first reported in 1930 by Doege and Potter [5,6]. Most case series on pleural fibrous tumours report hypoglycemia in about 2-4\% [7]. Doege Potter syndrome is more common in females (3 times) than males, if the size of the tumour is $>10 \mathrm{~cm}$ and if the tumour is in right hemithorax [8]. 
IGF family of proteins including IGF-1 and IGF-2 have been shown to have a role in many cellular activities.

Dysfunction of its regulatory functions may result in neoplstic transformation [3]. Alternatively, regulatory dysfunction may be an end result of neoplastic transformation where these proteins may be over-expressed [9]. IGF2 in Doege Potter syndrome has a larger molecular weight [10], with decreased binding ability to serum binding proteins. The usual IGF-2 assay results are frequently in the normal range and assays for big IGF-2 are not commercially available. Hence, the ratio IGF-2 to IGF-1 is used as a surrogate marker to diagnose Doege Potter syndrome [4].

The clinical presentation of both benign and malignant SFTP are usually comparable and local recurrences can occur in both. Surgical resection is the treatment modality of choice for both original and recurrent benign and malignant tumours [11], which cures hypoglycemia. Other treatment option involves chemotherapy. Treatment for hypoglycemic attacks include scheduled snacks, nocturnal or continous dextrose infusion or enteral tube feeding, corticosteroids, continous glucagon infusion [12].

\section{Figures}

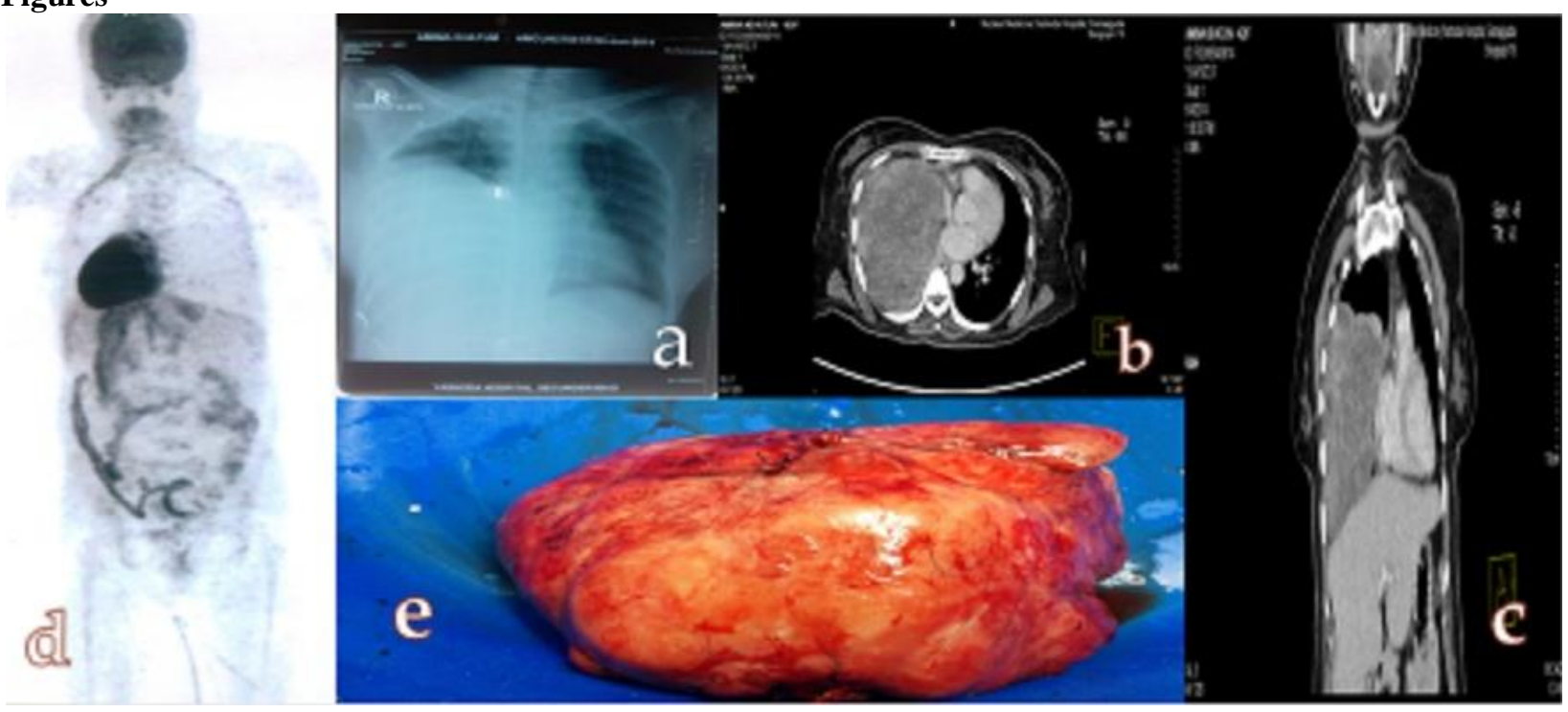

a) Chest $x$ ray---large soft tissue mass lesion involving right midzone and lower zone with obscured costophrenic angles, causing mediastinal shift to left side.

b \& c) Computed tomographic scan of the chest showing a giant mass involving most of right thoracic cavity with mass effect.

d) PET SCAN showing metabolically active mass in right lung

e) Resected fibrous tumor.

\section{Conclusion:-}

Doege Potter syndrome should be considered in patients who have intra-thoracic tumour and recurrent hypoinsulinemic hypoglycemia. Ratio of IGF-2 to IGF-1 is important to diagnose the syndrome than absolute IGF-2 levels. Surgery is the treatment of choice, with complete resection necessary to prevent recurrence. Identification of this paraneoplastic syndrome and adequate treatment can abolish life-threatening hypoglycemic episodes. However, regular follow-up may be necessary for any recurrences. 


\section{References:-}

1. Furukawa N, Hansky B, Niedermeyer J, Gummert J, Renner A: A silent gigantic solitary fibrous tumor of the pleura: case report. J Cardiothorac Surg 2011, 6:122.

2. Kalebi AY, Hale MJ, Wong ML, Hoffman T, Murray J: Surgically cured hypoglycemia secondary to pleural solitary fibrous tumour: case report and update review on the Doege-Potter syndrome. J Cardiothorac Surg 2009, 4:45.

3. Macauly VM. Insulin like growth factors and cancer. Br J Cancer. 1992; 65: 311-320.

4. Marks V, Teale JD: Tumours producing hypoglycaemia. Endocrine-Related Cancer 1998, 5:111-129.

5. Doege KW. Fibrosarcoma of the mediastinum. Ann Surg 1930; 92: 955-961.

6. Potter RP., Intrathoracic Tumours. Case Report. Radiology 1930; 14; 60-61.

7. Cardillo G., Facciolo F., Cavazzana A.O., Capece G., Gasparri R., and Martelli M. Localised (solitary) fibrous tumours of the pleura: an analysis of 55 patients. Ann Thorac Surg 2000; 70 (6): 1808-1812.

8. England D. M., Hochholzer L., and McCarthy M. J. Localised Benign and Malignant Fibrous Pleural Tumours of the pleura. A clinicopathologic review of 223 cases. Am J Surg Pathol 1989; 13: 8: 640-658.

9. Kondo M., Suzuki H., Ueda R., Osada H., Takagi K., Takahashi Toshitada., and Takahashi T. Frequent loss of imprinting of the H19 gene is often associated with its over-expression in lung cancers. Oncogene 1995; 10: 1193-1198.

10. Fukuda I., Hizunka N., Takano K., Asakawa-Yasumoto K., Shizume K., Demura H. Characterization of IGF-2 and IGF binding proteins in patients with Non Islet Cell tumour hypoglycemia. Endocrine Journal 1993, 40 (1), 111-119.

11. Santos RS, Haddad R, Lima CE, Liu YL, Misztal M, Ferreira T, Boasquevisque CH, Luketich JD, Landreneau RJ: Patterns of recurrence and long-term survival after curative resection of localized fibrous tumors of the pleura. Clin Lung Cancer 2005, 7:197-201.

12. Schutt et al: Doege-Potter syndrome presenting with hypoinsulinemic hypoglycemia in a patient with a malignant extrapleural solitary fibrous tumor: a case report; Journal of Medical Case Reports 2013, 7:11. 